Oncology

\title{
Evolution of the International Sarcoma Community: A Personal Perspective
}

\author{
George D. Demetri \\ Dana-Farber Cancer Institute, Ludwig Center at Harvard, Harvard Medical School, Boston, MA, USA
}

Patient care and research related to sarcoma is a uniquely positive and a globally collaborative environment. In contrast to many other fields of science, the international sarcoma community works together towards a common goal, thus enhancing our ability to achieve meaningful milestones in clinical research and disease management. The international sarcoma community shares a common interest in improving the lives of individuals with these rare and heterogeneous forms of cancer. However, in the face of finite resources for funding research and the low incidence of sarcomas relative to that of many other common diseases, governments, industry and philanthropists might question whether there is sufficient return on investment to justify investing in sarcoma research.

Undeniably, there are several good reasons for studying rare forms of cancer such as sarcomas.

Ethical Obligation: Sarcomas disproportionately affect children and young people who have long and productive lives ahead of them.

Practical Science: Sarcomas offer opportunity to learn something uniquely powerful about human tumour biology by providing clues about oncogenic drivers and identifying novel targets, which may also have an impact in other more common forms of human disease.

Utilitarian Critique: Although it stands to reason that resources could be invested in research targeting more

\section{KARGER}

() 2018 S. Karger AG, Basel

E-Mail karger@karger.com

www.karger.com/ocl common diseases than sarcoma to benefit greater numbers of people, there may be a comparative advantage to testing new treatments in sarcoma. To profit from this advantage, the scientific evidence generated by sarcoma research must be more accurate and the patient samples must be more homogenous. The international sarcoma community is getting increasingly proficient at designing more effective studies that lead to practice-changing outcomes for patient benefit.

\section{Review of Historical Sarcoma Landmarks}

In the 1970s, government-funded clinical trials conducted by the National Cancer Institute and the European Organisation for Research and Treatment of Cancer provided proof-of-concept evidence for solid tumour anticancer activity. In the early years of chemotherapy, a phase II evaluation of adriamycin (doxorubicin) in human neoplasia attempted to identify cancers in which doxorubicin might be effective [1]. This "one size fits all" clinical trial included patients with various cancer types and observed best results in lymphomas, sarcomas and breast carcinoma. Thus, early on, sarcomas were demonstrated as a proving ground for the efficacy of chemotherapy. The study reported 21 remissions in 64 sarcoma patients and this comprised 5 out of 9 osteogenic sarco- 
mas, 3 out of 8 leiomyosarcomas, 2 out of 14 fibrosarcomas, 3 out of 11 rhabdomyosarcomas, 2 out of 7 Ewing's sarcomas, 2 out of 3 haemangiosarcomas, and one remission each in chondrosarcomas (1/3), liposarcomas $(1 / 3)$, haemangiopericytomas (1/2) and neuroepithelioma $(1 / 1)$. Sarcomas thus exemplified the complexity of cancer diversity, even within a rare subset of cancers.

In ensuing years, there was considerable controversy about the reliability and practical efficacy of chemotherapy, with a key debate focused on whether there was a role for toxic chemotherapy in the adjuvant setting aiming to cure younger patients with osteosarcomas. That controversy ended definitively after a randomised trial involving just 36 patients with high-grade osteosarcoma of the extremities showed the dramatic impact of intensive multi-agent adjuvant chemotherapy compared with observation alone [2]. Cures in osteosarcoma changed clinical practice by demonstrating that expert multidisciplinary teamwork and innovations in therapy are key drivers to proving hypotheses in clinical research and improving outcomes in everyday practice. The sarcoma community is continuously looking to define other areas in which a small focused study might be sufficiently conclusive to prove the benefits of new therapies and provide reliable, high-quality evidence to serve as the basis for patient care recommendations.

The 1980s and early 1990s were a difficult time in the sarcoma field with many groups reporting that the magnitude of benefit of chemotherapy for soft tissue sarcomas (STS) was less than that for osteosarcomas [3-5]. By this time, it was clear that new agents and larger multicentre, international collaborations were required to improve patient outcomes in sarcoma [6].

\section{International Collaboration in the Sarcoma Field}

In 1993, a wealthy patient with sarcoma, who recognised that communication among sarcoma experts was suboptimal and was hindering progress in this field, organised a meeting in Florida for experts to discuss the state of knowledge about this group of rare diseases and share their individual expertise. The meeting proved to be the origin of the Connective Tissue Oncology Society which, in the years since, has grown into an organization of more than 400 members representing more than 30 countries. The Society's goal is to advance the care of patients with connective tissue tumours and to disseminate knowledge of all aspects of sarcoma tumour biology, including basic and clinical research. The full story is available on the Connective Tissue Oncology Society website.
The implementation of cross-centre and cross-country research projects brings together "dream teams" of sarcoma experts and is an important stimulus for ongoing collaboration. Occasionally, drug development can also serve as a stimulus for collaboration. In the 1990s, ecteinascidin-743 (ET-743), which eventually became known as trabectedin, was derived from the marine ascidian Ecteinascidia turbinata. Interest in ET-743 was considerable as laboratory studies had shown that this agent was effective against sarcomas even at exceptionally low doses [7]. Although American and European experts shared some concerns about the use of ET-743 in humans due to side effects such as severe thrombocytopenia and neutropenia [8], these concerns were countered by interesting data emerging about the unique sensitivity of sarcoma cell types to this agent [9]. Research eventually developed ways to deliver ET-743 to patients with improved safety, which allowed the development program to continue. A study that demonstrated the antitumour activity of ET-743 with acceptable toxicity in heavily pretreated patients with advanced STS or bone sarcoma was received with great interest [10].

Subsequently, mutually confirmatory phase II trials conducted in Europe and the United States further defined the activity of trabectedin in STS [11, 12]. These studies were performed in the early 2000s before the introduction of targeted therapies for any STS subtype other than gastrointestinal stromal tumours. As clinical research has since highlighted the scarcity of highly effective, single-gene drivers in most sarcomas, it appears likely that cytotoxic agents will continue to be a key part of the therapeutic arsenal to treat patients with the majority of sarcoma subtypes. The US investigators concluded that their results with trabectedin concurred with those of European studies, with rather marginal objective response rates in the range of only $10 \%$, although this should not be interpreted as evidence for a lack of efficacy in sarcoma, since a significant subset of patients can achieve durable disease control. Trabectedin was shown to induce a much higher response rate specifically against the myxoid/round cell subtype of liposarcomas, which is characterised by a reciprocal translocation between chromosomes 12 and $16(t[12 ; 16])$, and was associated with a response rate of $30 \%$ in liposarcomas overall. The authors suggested that the mechanism of action of trabectedin might relate to its binding to the DNA minor groove, thereby displacing the chimeric fusion transcript, which likely acts as an aberrant transcriptional regulator [12]. A subsequent retrospective evaluation of the efficacy of trabectedin in advanced pretreated myxoid/round cell lipo- 
sarcoma confirmed a high rate of antitumour activity in this histotype [13]. This study, which involved investigators in 5 countries (France, Italy, Spain, US, UK), was also a model of international collaboration and data sharing in the sarcoma field.

\section{Understanding Tumour Diversity is Key to Appropriate Therapy}

In the early 2000s, it became apparent that the traditional clinically-defined classification of sarcoma into STS (90\% of cases) and bone sarcomas (10\%) was no longer adequate to manage tumour diversity for trials of mechanism-targeting therapies. In 2011, a prospective epidemiological study with central pathology review and molecular testing provided a population-based data set supporting the heterogeneity of histological STS subtypes and identifying the relative incidence of most subsets [14]. At present, there are more than 100 histologicallydefined STS subtypes and, with genomically-defined subtypes, there are likely more than 1,000 such subsets.

Prior to 2016, only 20\% of STS subtypes had US Food and Drug Administration-approved molecular-targeted agents, represented by gastrointestinal stromal tumours (18\%) and dermatofibrosarcoma protuberans (2\%). However, with the introduction in 2016 of olaratumab, which targets the normal, non-mutated platelet-derived growth factor receptor, the proportion of STS subtypes with Food and Drug Administration-approved molecular-targeted agents is approaching $100 \%$, defined by those subsets for which doxorubicin is considered an effective agent. In a phase II trial, olaratumab in combination with doxorubicin produced a highly significant 11.8 months advantage in median overall survival over doxorubicin alone (26.5 vs. 14.7 months, hazard ratio $0.46 ; 95 \%$ CI $0.30-0.71 ; p=0.0003$ ) [15]. If the efficacy of this combination is confirmed in the phase III ANNOUNCE study (ClinicalTrial.gov identifier: NCT02451943) [16], it represents a major opportunity for the sarcoma community to identify other pairings of targeted therapy with chemotherapeutic agents.

\section{Conclusions}

The sarcoma field has advanced far beyond anthracyclines and ifosfamide, and arguments over the relative dose intensity of these 2 agents, as the sole options for treating STS. Other "repurposed" chemotherapy regi-

mens, such as gemcitabine and docetaxel, also show activity in several sarcoma subtypes. Trabectedin provides benefit to a wide range of patients with advanced STS, with a particular impact in leiomyosarcomas and liposarcomas. Targeted therapy and immune-oncology therapy are providing important insights into oncogenic drivers and mechanisms of resistance and offer opportunity for novel pairings with other agents. In this unique environment of many sarcoma subtypes and many new therapies, sarcoma patients more than ever need experts to define the best options, best sequences and best new approaches based on high-quality science and effective collaborations. The international sarcoma community takes great pride in being widely respected as one that is highly and uniquely collaborative in the interests of the patients we serve.

\section{Acknowledgements}

Writing assistance was provided by Content Ed Net (Madrid, Spain) with funding from PharmaMar, Madrid, Spain.

\section{Disclosure Statement}

G.D.D. has received consultancy fees from Adaptimmune, Blueprint Medicines, Caris Life Sciences, Daiichi-Sankyo, Eisai, EMD-Serono, Janssen Oncology, Kolltan Pharmaceuticals, Novartis, and PharmaMar; has received research support from Bayer, Janssen Oncology, Novartis, and Pfizer; is a Member of the Board of Directors and Scientific Advisory Board for Blueprint Medicines, and Merrimack Pharmaceuticals; is a consultant on Scientific Advisory Boards for Caris Life Sciences, and Daiichi-Sankyo; and has equity in Blueprint Medicines, Caris Life Sciences, G1 Therapeutics, and Merrimack Pharmaceuticals.

The author has no other relevant affiliations or financial involvement with any organization or entity with a financial interest in or financial conflict with the subject matter or materials discussed in the manuscript apart from those disclosed.

References

Oncology 2018;95(suppl 1):1-4 DOI: $10.1159 / 000494859$
1 O'Bryan RM, Luce JK, Talley RW, Gottlieb JA, Baker LH, Bonadonna G. Phase II evaluation of adriamycin in human neoplasia. Cancer. 1973 Jul;32(1):1-8.

2 Link MP, Goorin AM, Miser AW, Green AA, Pratt CB, Belasco JB, et al. The effect of adjuvant chemotherapy on relapse-free survival in patients with osteosarcoma of the extremity. N Engl J Med. 1986 Jun;314(25):1600-6.

3 Antman K, Crowley J, Balcerzak SP, Rivkin SE, Weiss GR, Elias A, et al. An intergroup phase III randomized study of doxorubicin and dacarbazine with or without ifosfamide and mesna in advanced soft tissue and bone sarcomas. Clin Oncol. 1993 Jul;11(7):1276-85. 
4 Edmonson JH, Ryan LM, Blum RH, Brooks JS, Shiraki M, Frytak S, et al. Randomized comparison of doxorubicin alone versus ifosfamide plus doxorubicin or mitomycin, doxorubicin, and cisplatin against advanced soft tissue sarcomas. J Clin Oncol. 1993 Jul;11(7): 1269-75.

5 Santoro A, Tursz T, Mouridsen H, Verweij J, Steward W, Somers R, et al. Doxorubicin versus CYVADIC versus doxorubicin plus ifosfamide in first-line treatment of advanced soft tissue sarcomas: a randomized study of the European Organization for Research and Treatment of Cancer Soft Tissue and Bone Sarcoma Group. J Clin Oncol. 1995 Jul;13(7): 1537-45.

6 Santoro A. Advanced soft tissue sarcoma: how many more trials with anthracyclines and ifosfamide? Ann Oncol. 1999 Feb;10(2): $151-4$.

7 Izbicka E, Lawrence R, Raymond E, Eckhardt G, Faircloth G, Jimeno J, et al. In vitro antitumor activity of the novel marine agent, ecteinascidin-743 (ET-743, NSC-648766) against human tumors explanted from patients. Ann Oncol. 1998 Sep;9(9):981-7.
8 van Kesteren C, Cvitkovic E, Taamma A, López-Lázaro L, Jimeno JM, Guzman C, Math t RA, Schellens JH, Misset JL, Brain E, Hillebrand MJ, Rosing H, Beijnen JH. Pharmacokinetics and pharmacodynamics of the novel marine-derived anticancer agent ecteinascidin 743 in a phase I dose-finding study. Clin Cancer Res. 2000 Dec;6(12):4725-4732.

9 Li WW, Takahashi N, Jhanwar S, CordonCardo C, Elisseyeff Y, Jimeno J, Faircloth G, Bertino JR. Sensitivity of soft tissue sarcoma cell lines to chemotherapeutic agents: identification of ecteinascidin-743 as a potent cytotoxic agent. Clin Cancer Res. 2001;7:29082911.

10 Delaloge S, Yovine A, Taamma A, Riofrio M, Brain E, Raymond E, et al. Ecteinascidin-743: a marine-derived compound in advanced, pretreated sarcoma patients-preliminary evidence of activity. J Clin Oncol. 2001 Mar; 19(5):1248-55.

11 Yovine A, Riofrio M, Blay JY, Brain E, Alexandre J, Kahatt C, et al. Phase II study of ecteinascidin-743 in advanced pretreated soft tissue sarcoma patients. J Clin Oncol. 2004 Mar;22(5):890-9.
12 Garcia-Carbonero R, Supko JG, Manola J, Seiden MV, Harmon D, Ryan DP, et al. Phase II and pharmacokinetic study of ecteinascidin 743 in patients with progressive sarcomas of soft tissues refractory to chemotherapy. J Clin Oncol. 2004 Apr;22(8):1480-90.

13 Grosso F, Jones RL, Demetri GD, Judson IR, Blay JY, Le Cesne A, et al. Efficacy of trabectedin (ecteinascidin-743) in advanced pretreated myxoid liposarcomas: a retrospective study. Lancet Oncol. 2007 Jul;8(7):595-602.

14 Ducimetière F, Lurkin A, Ranchère-Vince $D$, Decouvelaere AV, Péoc'h M, Istier L, et al. Incidence of sarcoma histotypes and molecular subtypes in a prospective epidemiological study with central pathology review and molecular testing. PLoS One. 2011;6(8):e20294.

15 Tap WD, Jones RL, Van Tine BA, Chmielowski B, Elias AD, Adkins D, et al. Olaratumab and doxorubicin versus doxorubicin alone for treatment of soft-tissue sarcoma: an open-label phase $1 \mathrm{~b}$ and randomised phase 2 trial. Lancet. 2016 Jul;388(10043):488-97.

16 ClinicalTrials.gov. A randomized, doubleblind, placebo-controlled, phase 3 trial of doxorubicin plus olaratumab versus doxorubicin plus placebo in patients with advanced or metastatic soft tissue sarcoma. https://clinicaltrials.gov/ct2/show/NCT02451943. 